\title{
Body mass index bias in defining obesity of diverse young adults: the Training Intervention and Genetics of Exercise Response (TIGER) Study
}

\author{
Andrew S. Jackson ${ }^{1}$, Kenneth J. Ellis ${ }^{2}$, Brian K. McFarlin ${ }^{1}$, Mary H. Sailors ${ }^{2}$ and Molly S. Bray ${ }^{2}$ \\ ${ }^{1}$ Department of Health and Human Performance, The University of Houston, 3855 Holman Street, Houston, TX 77204-6015, USA \\ ${ }^{2}$ USDA/ARS Children's Nutrition Research Center, Baylor College of Medicine, Houston, TX, USA
}

(Received 20 October 2008 - Revised 29 January 2009 - Accepted 5 March 2009 - First published online 6 April 2009)

The BMI cut-score used to define overweight and obesity was derived primarily using data from Caucasian men and women. The present study evaluated the racial/ethnic bias of BMI to estimate the adiposity of young men and women (aged 17-35 years) using dual-energy X-ray absorptiometry (DXA) determination of percentage body fat (DXA-BF\%) as the referent standard. The samples were 806 women and 509 men who were tested from one to three times over 9 months providing 1300 observations for women and 820 observations for men. Linear mixed models (LMM) regression showed that with age and BMI controlled, DXA-BF\% of African-American (AA) men and women, Asian-Indian men and women, Hispanic women and Asian women significantly differed from non-Hispanic white (NHW) men and women. For the same BMI of NHW women, the DXA-BF\% of AA women was $1.76 \%$ lower, but higher for Hispanic (1.65\%), Asian (2.65\%) and Asian-Indian (5.98\%) women. For the same BMI of NHW men, DXA-BF\% of AA men was $4.59 \%$ lower and $4.29 \%$ higher for Asian-Indian men. Using the recommended BMI cut-scores to define overweight and obesity systematically overestimated overweight and obesity prevalence for AA men and women, and underestimated prevalence for Asian-Indian men and women, Asian women and Hispanic women. The present study extends the generalisability of research documenting the racial/ethnic bias of the universal overweight and obesity BMI cut-scores.

Percentage body fat: BMI: Overweight: Obesity: Diversity

BMI, the ratio of weight and height, is an accepted public health method to define overweight and obesity ${ }^{(1,2)}$. In an invited commentary to the British Journal of Nutrition, Deurenberg stated his opposition to using a universal BMI cutscore to define obesity ${ }^{(3)}$. He maintained that the practice of defining obesity with a BMI of $30 \mathrm{~kg} / \mathrm{m}^{2}$ was based on data from Caucasian men and women from Europe and the USA and represented a percentage body fat $(\mathrm{BF} \%)$ of about $25 \%$ for young Caucasian men and $35 \%$ for young Caucasian women $^{(4)}$. Evidence supporting Deurenberg's position comes from research documenting age, sex and race/ethnic group bias in predicting $\mathrm{BF} \%$ from $\mathrm{BMI}^{(5-13)}$. These studies show that the $\mathrm{BF} \%$ for BMI systematically varies due to age, sex and race/ethnic group.

Obesity has become a pandemic in the USA and the American population is growing in ethnic and racial diversity. Data from the National Health and Nutrition Examination Survey (NHANES) $2003-4^{(14)}$ show that the percentage of all Americans over the age of 20 years who exceeded the overweight BMI standard of $\geq 25 \mathrm{~kg} / \mathrm{m}^{2}$ was $66.3 \%$, while $30.5 \%$ were considered obese $\left(\mathrm{BMI} \geq 30 \mathrm{~kg} / \mathrm{m}^{2}\right)$. These high prevalence levels are linked to overweight and obesity in young adulthood. The NHANES data documented that $57.1 \%$ of young adults (aged 20-39 years) were overweight, while obesity among this group approaches $30 \%^{(14)}$. The prevalence of overweight and obesity of African-American (AA) and Mexican American men and women aged 20-39 years is higher than non-Hispanic white (NHW) men and women, linking the prevalence not only to sex, but also racial/ethnic group.

Recent NHANES data ${ }^{(15,16)}$ showed that excess deaths were associated with obesity and underweight, but not overweight. Fontaine et al. ${ }^{(17)}$ reported that the association between longevity and BMI of AA and NHW adults differed. The optimal BMI associated with longevity was higher for AA men and women than NHW men and women. Stevens et al. ${ }^{(18)}$ reported that BMI was a less potent risk factor in AA women than NHW women when educational status was controlled. Manson \& Bassuk $^{(19)}$ report that the ethnic difference in longevity associated with a BMI may in part be due to race bias of BMI to estimate adiposity. The present study was designed to evaluate the fidelity of BMI to estimate adiposity of racially/ethnically diverse young men and women. The goal was to examine the race/ethnic group effect on the BMI prediction of $\mathrm{BF} \%$ using dual-energy X-ray absorptiometry (DXA) determination of BF\% (DXA-BF\%) as the referent adiposity standard.

Abbreviations: AA, African-American; BF\%, percentage body fat; DXA, dual-energy X-ray absorptiometry; DXA-BF\%, dual-energy X-ray absorptiometry determination of body fat percentage; LMM, linear mixed model; NHANES, National Health and Nutrition Examination Survey; NHW, non-Hispanic white; TIGER, Training Intervention and Genetics of Exercise Response.

* Corresponding author: Dr Andrew S. Jackson, fax + 1713743 9860, email udde@mac.com 


\section{Methods}

\section{Subjects}

Subjects were recruited from the Training Intervention and Genetics of Exercise Response (TIGER) study. The TIGER subjects were students enrolled at the University of Houston (Houston, TX, USA). The target subject was a sedentary individual under the age of 35 years who exercised less than $30 \mathrm{~min} /$ week for the previous 6 months, and was not actively limiting energy intake by dietary modification. Subjects were excluded from the study if they had a physical or physiological contraindication to aerobic exercise, a known metabolic disorder that may alter body composition, or were pregnant or lactating.

The TIGER study subjects engaged in 30 weeks (two semesters) of exercise training, $3 \mathrm{~d} /$ week for $30 \mathrm{~min} / \mathrm{d}$ at $65-85 \%$ of heart rate-defined $\mathrm{VO}_{2 \max }$. The data came from five yearly cohorts. The sample consisted of 806 females and 509 males who ranged in age from 17 to 35 years. These data included men and women who had from one to three measurement visits over the 9-month study duration. The measurement visits were at baseline, after 15 weeks, and at the end of the 30-week exercise programme. The total number of observations for the visits was 1300 for women and 820 for men. The race/ethnic group composition of the male and female samples differed slightly. The composition of the 509 men at baseline was: NHW, $37 \%$; Hispanic, 26\%; AA, 22\%; Asian Indian, 5\%; Asian, $10 \%$. The composition of the 806 women at baseline was: NHW, $29 \%$; Hispanic, $25 \%$; AA, $35 \%$; Asian-Indian, $3 \%$; Asian, $8 \%$.

\section{Measurements}

Height was determined with a stadiometer (SECA Road Rod; SECA, Hanover, MD, USA) and weight was measured with a digital scale (SECA 770). Subjects reported their birth date, sex and race/ethnicity using a standard, coded self-report demographic form. DXA was used to measure BF\%. The DXA data for the first two cohorts were only measured at baseline and 30-week time points. DXA data were available for all three test visits for cohorts 3 to 5 if the subject completed the exercise programme. All individuals had baseline data. The number of individuals who had repeat tests were: 15 weeks, 311 women and 217 men; 30 weeks, 183 women and ninety-four men.

Whole-body DXA scans were completed on a Hologic Delphi-A unit (adult whole body software v. 11.2; Hologic, Inc., Bedford, MA, USA) and a Hologic Discovery W instrument (adult whole body software QDR $v$. 12.3). The same trained technicians administered the DXA scans. The instruments were calibrated daily with a spine standard and weekly with a step calibrator, as described by the manufacturer. All female participants completed a standard urine pregnancy test before DXA testing to ensure that they were not pregnant before scanning. Subjects were asked to lie in the supine position and remain still. The entire scan was completed in less than $6 \mathrm{~min}$. Software supplied from the manufacturer was used to calculate whole-body (minus the head) fat mass, lean mass and bone mineral mass. Total DXA weight was computed by summing the DXA parts and used to compute DXA-BF\%. As recommended by Lohman \& Chen ${ }^{(20)}$, DXA weight and scale-measured body weight were compared with linear regression. The $R^{2}$ between measured and DXA weight was $>0.99$. The slope of the measuredDXA weight regression line of 1.01 (95\% CI 1.00, 1.02) and the intercept of -0.08 (95\% CI $-0.37,0.20)$ were within chance variation of 1.0 and 0 . The standard error of the estimate for scale-measured weight was $1.5 \mathrm{~kg}$.

\section{Statistical methods}

Maximum likelihood, linear mixed models (LMM) regression was used to model the data with a random intercept growth model $^{(21)}$. A major advantage of LMM is that all data can be used with subjects who have a different number of observations over time ${ }^{(21,22)}$. Stata version 10 (xtmixed program) was used for data analysis ${ }^{(23)}$. The first LMM examined the linearity of the BMI and DXA-BF\% relationship. The second model examined the effect of race/ethnicity group on DXA-BF\% with age and BMI statistically controlled. The dependent variable for the LMM analyses was DXA-BF\% and the fixed effects variables were BMI and race/ethnic group, which was dummy coded using NHW women and men as the referent groups. The repeat tests were the random part of the model. Each fixed effect LMM regression coefficient was tested with a $z$-test to determine if it was significantly different from zero ${ }^{(23)}$. A log-ratio test ${ }^{(21-23)}$ was used to determine if the model that included race/ethnic group provided a better fit of the data than the BMI model. It has been previously documented ${ }^{(13)}$ that for the same $\mathrm{BMI}$, the $\mathrm{BF} \%$ of women is $\geq 10.4 \%$ higher than that of men, and that age accounts for BF\% independently of BMI and sex. For these reasons, the men and women's data were analysed separately and age was used as a covariate.

\section{Results}

Table 1 gives the descriptive statistics for the women and men and the number of observations for the total sample and for race/ethnicity group. The women and men were similar in age and the data exhibited the well-documented sex differences in physical characteristics for height, weight and body composition. The NHW women were significantly taller than Hispanic, Asian-Indian and Asian women and heavier than Asian-Indian and Asian women, while AA women were heavier than NHW women. Compared with NHW women, the BMI of Hispanic $(P=0.057)$ and AA women was higher and the BMI for Asian women was lower. The DXA bone mineral mass and lean mass of all race/ethnic groups were significantly different from NHW women. The DXA-BF\% of Hispanic and Asian-Indian women was significantly higher than that of NHW women, while DXA-BF\% did not differ between NHW, AA and Asian women. The NHW men were significantly taller than Hispanic, Asian-Indian and Asian men. There were no significant race/ethnic group differences for weight or BMI in males. The DXA bone mineral and lean mass of all groups were significantly different from those of the NHW men. The mean DXA fat mass of AA men was significantly $(P=0.023)$ lower than that of NHW men. The mean DXA-BF\% of AA men was $3.5 \%$ lower than that for NHW men.

The LMM analyses are provided in Table 2 (women) and Table 3 (men). Provided are the LMM regression coefficients (estimates), the standard errors of the coefficient (SE) and the 
Table 1. Characteristics of the Training Intervention and Genetics of Exercise Response (TIGER) women ( $n$ 806) and men ( $n$ 509) for the total sample and contrasted by race/ethnic groups

(Mean values and standard deviations)

\begin{tabular}{|c|c|c|c|c|c|c|c|c|c|c|c|c|}
\hline \multirow[b]{2}{*}{ Variable } & \multicolumn{2}{|c|}{ White } & \multicolumn{2}{|c|}{ Hispanic } & \multicolumn{2}{|c|}{$\begin{array}{l}\text { African- } \\
\text { American }\end{array}$} & \multicolumn{2}{|c|}{ Asian-Indian } & \multicolumn{2}{|c|}{ Asian } & \multicolumn{2}{|c|}{ Total } \\
\hline & Mean & SD & Mean & SD & Mean & SD & Mean & SD & Mean & SD & Mean & SD \\
\hline \multicolumn{13}{|l|}{ TIGER women } \\
\hline Observations & \multicolumn{2}{|c|}{379} & \multicolumn{2}{|c|}{325} & \multicolumn{2}{|c|}{449} & \multicolumn{2}{|c|}{43} & \multicolumn{2}{|c|}{104} & \multicolumn{2}{|c|}{1300} \\
\hline Age (years) & $21 \cdot 7$ & $3 \cdot 1$ & 21.6 & 2.9 & $21 \cdot 1$ & 3.2 & 21.3 & $1 \cdot 7$ & $21 \cdot 1$ & $2 \cdot 2$ & 21.4 & 3.0 \\
\hline Height $(\mathrm{cm})$ & $164 \cdot 4$ & 6.5 & $159 \cdot 2^{*}$ & 5.8 & $164 \cdot 2$ & $6 \cdot 6$ & $155 \cdot 7^{*}$ & $6 \cdot 3$ & $156 \cdot 5^{\star}$ & $5 \cdot 6$ & $162 \cdot 1$ & $7 \cdot 0$ \\
\hline Weight (kg) & $68 \cdot 1$ & $16 \cdot 6$ & $67 \cdot 3$ & $15 \cdot 8$ & $73 \cdot 2^{*}$ & $19 \cdot 6$ & $57 \cdot 8^{*}$ & $12 \cdot 0$ & $55 \cdot 4^{*}$ & 11.5 & $68 \cdot 3$ & $17 \cdot 8$ \\
\hline BMI $\left(\mathrm{kg} / \mathrm{m}^{2}\right)$ & $25 \cdot 3$ & 5.9 & $26 \cdot 6$ & $6 \cdot 0$ & $27 \cdot 1^{*}$ & $6 \cdot 7$ & 23.9 & 4.5 & $22 \cdot 6^{*}$ & 3.9 & $26 \cdot 0$ & $6 \cdot 2$ \\
\hline $\mathrm{BMC}(\mathrm{kg})$ & $2 \cdot 2$ & 0.3 & $2 \cdot 0^{*}$ & 0.3 & $2 \cdot 4^{*}$ & 0.3 & $1.9^{*}$ & 0.2 & $1.9^{*}$ & 0.2 & $2 \cdot 2$ & 0.4 \\
\hline Lean mass $(\mathrm{kg})$ & $43 \cdot 4$ & 6.9 & $40 \cdot 9^{*}$ & 6.5 & $46 \cdot 2^{*}$ & 8.2 & $34 \cdot 5^{\star}$ & $5 \cdot 8$ & $35 \cdot 8^{*}$ & $5 \cdot 6$ & $42 \cdot 8$ & 7.9 \\
\hline Fat mass $(\mathrm{kg})$ & 21.9 & $10 \cdot 8$ & $23 \cdot 7$ & $10 \cdot 0$ & 23.9 & $12 \cdot 0$ & $20 \cdot 8$ & $6 \cdot 8$ & $17 \cdot 1^{*}$ & $6 \cdot 8$ & $22 \cdot 6$ & $10 \cdot 8$ \\
\hline DXA-BF\% & $31 \cdot 0$ & 7.4 & $34 \cdot 3^{\star}$ & $6 \cdot 6$ & $31 \cdot 3$ & 7.9 & $35 \cdot 7^{*}$ & $5 \cdot 1$ & $30 \cdot 3$ & $6 \cdot 1$ & $32 \cdot 0$ & $7 \cdot 4$ \\
\hline \multicolumn{13}{|l|}{ TIGER men } \\
\hline Observations & \multicolumn{2}{|c|}{305} & \multicolumn{2}{|c|}{217} & \multicolumn{2}{|c|}{183} & \multicolumn{2}{|c|}{37} & \multicolumn{2}{|c|}{78} & \multicolumn{2}{|c|}{820} \\
\hline Age (years) & $22 \cdot 3$ & 3.4 & 21.6 & 2.5 & $21 \cdot 1^{\star}$ & 2.9 & $22 \cdot 7$ & 3.8 & $21 \cdot 7$ & $2 \cdot 2$ & $21 \cdot 8$ & 3.0 \\
\hline Height $(\mathrm{cm})$ & $177 \cdot 1$ & $6 \cdot 0$ & $173 \cdot 3^{\star}$ & $6 \cdot 6$ & $177 \cdot 6$ & 6.5 & $172 \cdot 3^{*}$ & 5.9 & $172 \cdot 2^{*}$ & $7 \cdot 2$ & $175 \cdot 5$ & 6.7 \\
\hline Weight (kg) & 83.6 & $18 \cdot 1$ & 82.0 & $18 \cdot 8$ & $85 \cdot 8$ & $20 \cdot 7$ & $76 \cdot 4$ & $19 \cdot 2$ & 82.9 & $25 \cdot 6$ & 83.3 & $19 \cdot 8$ \\
\hline BMI $\left(\mathrm{kg} / \mathrm{m}^{2}\right)$ & $26 \cdot 6$ & $5 \cdot 2$ & $27 \cdot 3$ & $5 \cdot 6$ & $27 \cdot 2$ & $5 \cdot 8$ & $25 \cdot 8$ & $5 \cdot 8$ & $27 \cdot 8$ & $7 \cdot 1$ & $27 \cdot 0$ & $5 \cdot 7$ \\
\hline $\mathrm{BMC}(\mathrm{kg})$ & $2 \cdot 8$ & 0.4 & $2 \cdot 7^{\star}$ & 0.4 & $3 \cdot 1^{*}$ & 0.5 & $2 \cdot 5^{\star}$ & 0.4 & $2 \cdot 6^{*}$ & 0.4 & $2 \cdot 8$ & 0.5 \\
\hline Lean mass $(\mathrm{kg})$ & $62 \cdot 0$ & $9 \cdot 3$ & $59 \cdot 9^{\star}$ & $10 \cdot 2$ & $65 \cdot 6^{*}$ & 9.5 & $53 \cdot 3^{*}$ & $8 \cdot 8$ & $59 \cdot 6^{*}$ & $12 \cdot 4$ & $61 \cdot 6$ & $10 \cdot 3$ \\
\hline Fat mass $(\mathrm{kg})$ & $17 \cdot 8$ & $10 \cdot 2$ & $18 \cdot 1$ & 9.8 & $15 \cdot 6^{\star}$ & $12 \cdot 4$ & $19 \cdot 2$ & $11 \cdot 2$ & $19 \cdot 4$ & $13 \cdot 6$ & $17 \cdot 6$ & $11 \cdot 1$ \\
\hline DXA-BF\% & $20 \cdot 3$ & 7.5 & 21.4 & 6.9 & $16 \cdot 8^{*}$ & $8 \cdot 3$ & $24 \cdot 1^{*}$ & 8.8 & $21 \cdot 7$ & 8.2 & $20 \cdot 1$ & 7.9 \\
\hline
\end{tabular}

$\mathrm{BMC}$, bone mineral content; DXA-BF\%, body fat percentage determined by dual-energy $\mathrm{X}$-ray absorptiometry.

${ }^{*}$ Mean value was significantly different from that of white men or women $(P<0.01)$.

probability $(P)$ that the coefficient was significantly different from 0. LMM I showed that, with age controlled, the BMI relationship with DXA-BF\% for men was linear, but quadratic for women. Fig. 1 gives the bivariate relationship for all observations contrasted by sex. Also provided in Fig. 1 are the quadratic and linear lines of best fit for the women and men's bivariate distributions. These graphs show that the DXA-BF\% of women was higher than that of men for the same BMI through the range studied.

LMM II in Tables 2 and 3 included race/ethnic group with BMI. The log-ratio test confirmed that LMM II provided a better fit for both women and men $(P<0.001)$. The women's LMM regression weights for all race/ethnic groups were significantly different from the referent group, NHW women.
For the same age and BMI, the DXA-BF\% of AA women was $1.76 \%$ lower than that of NHW women and the DXA$\mathrm{BF} \%$ of Hispanic, Asian-Indian and Asian women were significantly higher. The race/ethnic group bias for the same age and BMI ranged from $1.65 \%$ for Hispanic women to nearly $6 \%$ for Asian-Indian Women. The analysis of the men's data showed that the LMM II regression weights of Hispanic and Asian men were within random variation of NHW men. The LMM regression weights for AA and Asian-Indian men were significantly different from that of NHW men and the effects were substantial. For the same age and BMI, the DXA-BF\% of AA men was $4.57 \%$ lower than that of NHW men while the DXA-BF\% of Asian-Indian men was $4.29 \%$ higher. A final LMM analyses examined the race/ethnic

Table 2. Maximum likelihood linear mixed models (LMM) analyses of the Training Intervention and Genetics of Exercise Response (TIGER) women (1300 total observations)

(Estimates with their standard errors)

\begin{tabular}{|c|c|c|c|c|c|c|}
\hline \multirow[b]{2}{*}{ Term } & \multicolumn{3}{|c|}{ LMM I } & \multicolumn{3}{|c|}{ LMM II } \\
\hline & Estimate & SE & $P$ & Estimate & SE & $P$ \\
\hline \multicolumn{7}{|l|}{ Fixed part } \\
\hline Constant $(\beta)$ & 13.0535 & $2 \cdot 2726$ & $<0.001$ & -14.4900 & $2 \cdot 1190$ & $<0.001$ \\
\hline Age $(\beta)$ & 0.0219 & 0.0479 & 0.647 & 0.0080 & 0.0438 & 0.855 \\
\hline $\mathrm{BMI}(\beta)$ & $2 \cdot 3527$ & 0.1396 & $<0.001$ & 2.4274 & 0.1302 & $<0.001$ \\
\hline $\mathrm{BMI}^{2}(\beta)$ & -0.0230 & 0.0023 & $<0.001$ & -0.0236 & 0.0021 & $<0.001$ \\
\hline Hispanic $(\beta)$ & & & & 1.6520 & 0.3740 & $<0.001$ \\
\hline African-American ( $\beta$ ) & & & & -1.7619 & 0.3437 & $<0.001$ \\
\hline Asian-Indian $(\beta)$ & & & & 5.9779 & 0.7415 & $<0.001$ \\
\hline Asian $(\beta)$ & & & & $2 \cdot 6480$ & 0.5590 & $<0.001$ \\
\hline \multicolumn{7}{|l|}{ Random part } \\
\hline Intercept (SD) & $4 \cdot 18$ & 0.11 & & $3 \cdot 75$ & $0 \cdot 10$ & \\
\hline Error (SD) & $1 \cdot 13$ & 0.04 & & $1 \cdot 13$ & 0.04 & \\
\hline
\end{tabular}


Table 3. Maximum likelihood linear mixed models (LMM) analyses of the Training Intervention and Genetics of Exercise Response (TIGER) men (820 total observations)

(Estimates with their standard errors)

\begin{tabular}{|c|c|c|c|c|c|c|}
\hline \multirow[b]{2}{*}{ Term } & \multicolumn{3}{|c|}{ LMM I } & \multicolumn{3}{|c|}{ LMM II } \\
\hline & Estimate & SE & $P$ & Estimate & SE & $P$ \\
\hline \multicolumn{7}{|l|}{ Fixed part } \\
\hline Constant $(\beta)$ & $-11 \cdot 8441$ & 1.6975 & $<0.001$ & -9.4839 & 1.5855 & $<0.001$ \\
\hline Age $(\beta)$ & 0.0647 & 0.0698 & 0.353 & -0.0173 & 0.0641 & 0.787 \\
\hline BMI $(\beta)$ & $1 \cdot 1338$ & 0.0337 & $<0.001$ & 1.1427 & 0.0310 & $<0.001$ \\
\hline Hispanic $(\beta)$ & & & & -0.0707 & 0.5047 & 0.889 \\
\hline African-American $(\beta)$ & & & & -4.5940 & 0.5287 & $<0.001$ \\
\hline Asian-Indian $(\beta)$ & & & & 4.2932 & 0.9330 & $<0.001$ \\
\hline Asian $(\beta)$ & & & & 0.3004 & 0.7243 & 0.678 \\
\hline \multicolumn{7}{|l|}{ Random part } \\
\hline Intercept (SD) & 4.85 & $0 \cdot 16$ & & 4.33 & 0.14 & \\
\hline Error (SD) & $1 \cdot 19$ & 0.05 & & $1 \cdot 18$ & 0.05 & \\
\hline
\end{tabular}

group and BMI interaction. A log likelihood-ratio test confirmed that adding the $\mathrm{BMI} \times$ race/ethnic group interaction term for both men $(P=0.093)$ and women $(P=0.384)$ did not improve the fit over LMM II. This confirmed that the systematic race/ethnic bias was constant over the entire BMI range.

Figs. 2 and 3 show the significant ethnic group bias for women and men. The graphs include solid and dashed lines representing the NHW women and men DXA-BF\% for the BMI overweight and obesity cut-scores. These DXA-BF\% NHW cut-scores for overweight and obesity were: women, 31.2 and $36.9 \%$; men, 18.7 and $24.6 \%$. The overweight and obese lines in the figures show that the BMI for Hispanic, Asian and Asian-Indian women is lower than the NHW women values of 25 and $30 \mathrm{~kg} / \mathrm{m}^{2}$ while the BMI for AA women is higher. Figure 3 shows that the BMI of AA men is about $4 \mathrm{~kg} / \mathrm{m}^{2}$ lower than NHW men and the BMI cut-score for Asian-Indian men is nearly $4 \mathrm{~kg} / \mathrm{m}^{2}$ higher. Table 4 gives these race/ethnic group BMI specific cutscores $(95 \% \mathrm{CI})$ equated to the NHW DXA-BF\% values.

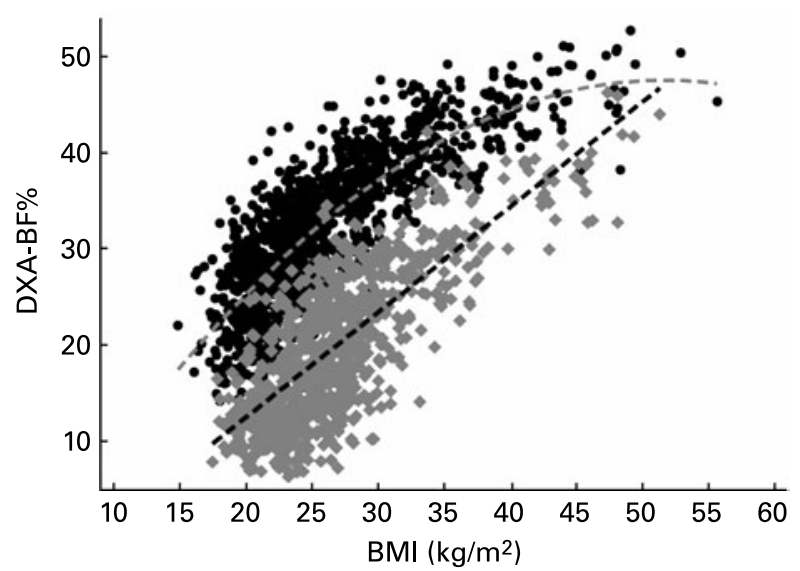

Fig. 1. The bivariate relationship between $\mathrm{BMI}$ and body fat percentage determined by dual-energy X-ray absorptiometry (DXA-BF\%) using 1300 total observations for women and 820 observations for men. (•), Women's data; $(\diamond)$, men's data. The relationship (regression line) for women was quadratic (---); for men it was linear (---).
The LMM III random model residuals were examined graphically two ways: normal curves superimposed on the histogram of residuals; standardised normal quartile probability plots of the residuals ${ }^{(21,23,24)}$. These graphs showed that the residuals were normally distributed (graphics not shown) with no serious outliers. The excellent fit of the LMM II data is further documented by the model error estimates provided in Tables 2 and 3 . The error estimates were $<1.2 \%$, which is close to the measurement error that would be expected when measuring BF\% with DXA technology.

\section{Discussion}

These TIGER results documented that the relationship between BMI and BF\% differs for men and women. Similar to results reported by other investigators ${ }^{(7,10,11,13)}$, these TIGER data found that, for a given BMI, the $\mathrm{BF} \%$ of women was higher than of men. These results with young adults documented that the relationship between BMI and $\mathrm{BF} \%$ of men was linear, but quadratic for women. For a BMI $<40 \mathrm{~kg} / \mathrm{m}^{2}$ the sex difference in $\mathrm{BF} \%$ was $\geq 10 \%$.

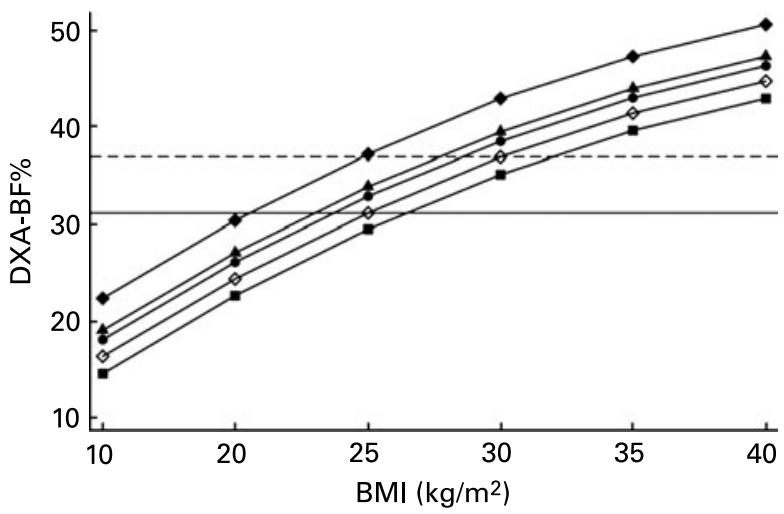

Fig. 2. Body fat percentage estimates determined by dual-energy X-ray absorptiometry (DXA-BF\%) for selected BMI values of women contrasted by race/ethnic groups. (-), DXA-BF\% value of $31.2 \%$, which equates to the BMI cut-score for overweight; (--), DXA-BF\% value of $36.9 \%$, which equates to the BMI cut-score for obese; $(\diamond)$, non-Hispanic whites; $(\bullet)$, Hispanic whites; $(\boldsymbol{\square})$, African-Americans; $(\bullet)$, Asian-Indians; $(\boldsymbol{\Delta})$, Asians. The graphs were constructed using linear mixed model (LMM) II to model the data (see Table 2). 


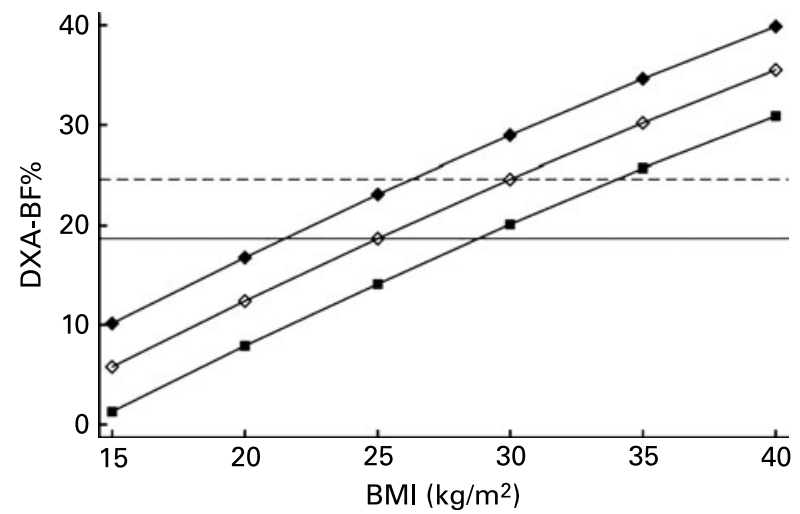

Fig. 3. Body fat percentage estimates determined by dual-energy X-ray absorptiometry (DXA-BF\%) for selected BMI values of men contrasted by non-Hispanic white $(\diamond)$, African-American $(\square)$ and Asian-Indian $(\diamond)$ groups. (-), DXA-BF\% value of $18.7 \%$, which equates to the BMI cut-score for overweight; (--), DXA-BF\% value of $24.6 \%$, which equates to the BMI cut-score for obese. The graphs were constructed using linear mixed model (LMM) II to model the data (see Table 3).

The non-linear relationship between $\mathrm{BMI}$ and $\mathrm{BF} \%$ has been documented with men and women from the Heritage Family Study ${ }^{(13)}$. The major difference between samples was that the Heritage study subjects ranged in age from 17 to 65 years, compared with the TIGER age range of 17 to 35 years, and the Heritage study $\mathrm{BF} \%$ was measured with a two-component method, not DXA.

An important finding of the present study was that BMI overestimated the DXA-BF\% of AA men and women. These TIGER results are consistent with the meta-analysis results reported by Deurenberg et al. ${ }^{(7)}$ and Jackson et al. ${ }^{(13)}$ but differed from the results reported by Gallagher et al. ${ }^{(11)}$. Deurenberg et al. found that using a BMI prediction equation derived with Caucasians overestimated the measured BF\% of AA men and women by nearly $2 \%$. The Heritage Family Study included 665 AA and NHW men and women who ranged in age from 17 to 65 years. In a second Heritage study $^{(25)}$ a race effect for abdominal visceral fat was found for both men and women. Controlling for age, BMI and waist circumference, the abdominal visceral fat measured by computed tomography of AA men and women was significantly lower than that of NHW men and women.

Other investigators have reported a race/ethnic group bias when estimating $\mathrm{BF} \%$ from $\mathrm{BMI}$ with equations derived from Caucasian men and women. Using samples of older Hispanic Americans and controlling for age, Fernandez et al. ${ }^{(9)}$ reported that for BMI values below $30 \mathrm{~kg} / \mathrm{m}^{2}$, Hispanic American women had a higher BF\% than either Caucasian or AA women, but, like these results, did not find a significant race/ethnic group effect for men. Several investigators ${ }^{(5-8)}$ reported that BMI systematically underestimated the $\mathrm{BF} \%$ of Asian subjects. Deurenberg-Yap et al. ${ }^{(8)}$ recommended that, for the same Caucasian BF\%, the obesity BMI cut-score for Chinese and Malays was $27 \mathrm{~kg} / \mathrm{m}^{2}$. These TIGER data showed that the obesity BMI cut-score for Asian women was $27.6(95 \%$ CI $26.6,28.9) \mathrm{kg} / \mathrm{m}^{2}$, within random variation of the Deurenberg-Yap et al. recommendation. DeurenbergYap et al. ${ }^{(8)}$ also reported a race bias for Singaporean Indians. Their data showed that, for the same BF\% of Caucasians, the BMI cut-score for obesity was $26 \mathrm{~kg} / \mathrm{m}^{2}$, which is within chance variation of the values we found with these young Asian-Indian men (26.5 (95\% CI 24.9, 28.1) kg/m $\left.{ }^{2}\right)$ and slightly higher than what we found for Asian-Indian women $\left(24.8(95 \%\right.$ CI $\left.23.6,25 \cdot 9) \mathrm{kg} / \mathrm{m}^{2}\right)$.

These TIGER results identified a significant Asian group effect for women, but not men, whereas Chang et al. ${ }^{(5)}$ and Deurenberg-Yap et al. ${ }^{(8)}$ reported a significant effect for both men and women. A likely reason for this difference was research design. Chang et al. and Deurenberg-Yap et al. combined the data of men and women and controlled for sex by including it as an independent variable of their multiple regression model. To examine this methodological issue, we combined the TIGER men and women's data and used sex as an independent variable (male $=0$, female $=1$ ). This LMM analysis (results not shown) revealed that, with age and sex statistically controlled, the Asian group effect was $1.72(P<0.001)$. This demonstrated that for the same BMI, the DXA-BF\% of Asian men and women would be $1.7 \%$ higher than that of NHW men and women. This suggested that the difference between studies was linked to combining men and women into the same statistical model. A strength of the present study was the large number of observations for men and women. This not only provided our sex-specific models with sufficient statistical power, but also controlled the effect of sex, which has been shown to be substantial. For the same BMI, the BF\% of women has consistently been found to be $\geq 10.4 \%$ higher than that of men ${ }^{(13)}$.

The recommended obesity cut-score of $30 \mathrm{~kg} / \mathrm{m}^{2(1,2)}$ was determined from the general trends in the relationship between

Table 4. Overweight and obese BMI cut-scores for race/ethnic groups equivalent to body fat percentage determined by dual-energy X-ray absorptiometry for 25 and $30 \mathrm{~kg} / \mathrm{m}^{2}$ of non-Hispanic white women and men (Mean values and $95 \%$ confidence intervals)

\begin{tabular}{|c|c|c|c|c|c|c|c|c|}
\hline \multirow[b]{3}{*}{ Group } & \multicolumn{4}{|c|}{ Women } & \multicolumn{4}{|c|}{ Men } \\
\hline & \multicolumn{2}{|c|}{ Overweight } & \multicolumn{2}{|c|}{ Obese } & \multicolumn{2}{|c|}{ Overweight } & \multicolumn{2}{|c|}{ Obese } \\
\hline & Mean & $95 \% \mathrm{Cl}$ & Mean & $95 \% \mathrm{Cl}$ & Mean & $95 \% \mathrm{Cl}$ & Mean & $95 \% \mathrm{Cl}$ \\
\hline African-American & $26 \cdot 4$ & $25 \cdot 9,27 \cdot 0$ & 31.9 & $31 \cdot 2,32 \cdot 5$ & $29 \cdot 0$ & $28 \cdot 2,29 \cdot 9$ & 34.2 & $33 \cdot 3,35 \cdot 2$ \\
\hline Asian-Indian & $20 \cdot 6$ & $19 \cdot 6,21 \cdot 6$ & $24 \cdot 8$ & $23 \cdot 6,25 \cdot 9$ & $21 \cdot 3$ & $19 \cdot 7,22 \cdot 9$ & $26 \cdot 5$ & $24 \cdot 9,28 \cdot 1$ \\
\hline Asian & $22 \cdot 9$ & $22 \cdot 2,23 \cdot 9$ & $27 \cdot 6$ & $26 \cdot 6,28 \cdot 9$ & $25 \cdot 0$ & & $30 \cdot 0$ & \\
\hline Hispanic & $23 \cdot 7$ & $23 \cdot 2,24 \cdot 3$ & 28.5 & $27 \cdot 8,29 \cdot 3$ & $25 \cdot 0$ & & $30 \cdot 0$ & \\
\hline White & $25 \cdot 0$ & & $30 \cdot 0$ & & $25 \cdot 0$ & & $30 \cdot 0$ & \\
\hline
\end{tabular}


BMI and morbidity and mortality rates, not on the accuracy of BMI to estimate BF\%. Recent NHANES results ${ }^{(15,16)}$ found that, compared with the normal-weight category (BMI 18.5 to $<25 \mathrm{~kg} / \mathrm{m}^{2}$ ), obesity (BMI $\geq 30 \mathrm{~kg} / \mathrm{m}^{2}$ ) was associated with excess mortality or CVD, whereas overweight (BMI 25 to $<30 \mathrm{~kg} / \mathrm{m}^{2}$ ) was not. Fontaine et al. ${ }^{(17)}$ reported that the BMI of young adults was related to longevity and the effect on AA men and women differed from that on NHW men and women. They reported that NHW men and women had a reduced longevity if their BMI was $\geq 30 \mathrm{~kg} / \mathrm{m}^{2}$ at age 20 to 30 years. The pattern for AA men and women differed. A consistent reduction in longevity did not take place with AA women until the BMI reached $\geq 37 \mathrm{~kg} / \mathrm{m}^{2}$ and $\geq 33 \mathrm{~kg} / \mathrm{m}^{2}$ for AA men. Stevens et al. ${ }^{(18)}$ reported that the reference $\mathrm{BMI}$ for a $40 \%$ higher risk of allcause mortality was $35.9 \mathrm{~kg} / \mathrm{m}^{2}$ in AA women compared with $27.3 \mathrm{~kg} / \mathrm{m}^{2}$ for NHW women. These TIGER results are consistent with the observation made by Manson \& Bassuk ${ }^{(19)}$ who stated that the ethnic variation in the relationship between BMI and total adiposity or visceral adiposity might partly account for the observed racial discrepancies reported by Fontaine et al. ${ }^{(17)}$.

A limitation of the present study is that the TIGER subjects are not a random sample of the US population, but the TIGER men and women are representative of the overweight and obesity prevalence of the national population of young adults ${ }^{(14)}$. The self-reported definition of racial/ethnic group membership was another limitation in the present study. The genetic technique of admixture analysis provides an objective method of defining racial heritage. Research has shown that admixture mapping cannot only localise, but also fine map, a phenotypically important variant ${ }^{(26)}$. The genetic examination of these TIGER data with admixture analysis is planned and will provide a clearer understanding of the source of the race/ethnic group bias found with these diverse young men and women.

These TIGER results support the findings of others ${ }^{(5-9,13,25)}$ that standardised obesity cut-points based on BMI do not represent equivalent levels of body fat across different race/ethnic groups. Our data indicate that, based on DXA-BF\%, overweight and obesity cut-points should be higher in AA men and women and lower in Asian-Indian, Asian and Hispanic women and Asian-Indian men. These findings document the inherent bias of using BMI as an indicator of obesity in diverse men and women. These results extend the generalisability of race/ethnic group bias of BMI to young adults. The large number of observations and separate models for women and men support the fidelity of these findings.

\section{Acknowledgements}

Support for the present study was provided by the National Institute of Diabetes \& Digestive \& Kidney Diseases/National Institutes of Health grant R01-DK062148 and by USDA/ARS contract 6250-51 000-046.

The present study was conducted according to the guidelines laid down in the Declaration of Helsinki and all procedures involving human subjects were approved by the institutional review committees on the use of human subjects for research at both the Baylor College of Medicine and the University of Houston, Houston, TX. Written informed consent was obtained from all subjects.
A. S. J. designed the study, performed the data analyses and drafted the paper. K. J. E. was responsible for all DXA measurements. B. K. M. contributed to the drafting of the paper and aided with DXA measurements. M. H. S. was responsible for database management and helped with data analysis. M. S. B. designed the TIGER study and contributed to drafting the manuscript.

The authors have no professional relationships with companies or manufacturers who may benefit from the results of the present study.

\section{References}

1. Kuczmarski RJ \& Flegal KM (2000) Criteria for definition of overweight in transition: background and recommendations for the United States. Am J Clin Nutr 72, 1075-1081.

2. World Health Organization (1998) Obesity: Preventing and Managing the Global Epidemic. Report of a WHO Consultation on Obesity. Geneva: WHO.

3. Deurenberg P (2001) Invited Commentary: Universal cut-off BMI points for obesity are not appropriate. $B r J$ Nutr 85, $135-136$

4. World Health Organization (1995) Physical Status: The Use and Interpretation of Anthropometry. WHO Technical Report Series no. 854. Geneva: WHO.

5. Chang CJ, Wu CH, Chang CS, et al. (2003) Low body mass index but high percent body fat in Taiwanese subjects: implications of obesity cutoffs. Int J Obes 27, 253-259.

6. Chung S, Song J, Shin H, et al. (2005) Korean and Caucasian overweight premenopausal women have different relationship of body mass index to percent body fat with age. J Appl Physiol 99, 103-107.

7. Deurenberg P, Yap M \& van Staveren WA (1998) Body mass index and percent body fat: a meta analysis among different ethnic groups. Int J Obes 22, 1164-1171.

8. Deurenberg-Yap M, Schmidt G, van Staveren WA, et al. (2000) The paradox of low body mass index and high body fat percentage among Chinese, Malays and Indians in Singapore. Int $J$ Obes 24, 1011-1017.

9. Fernandez JR, Heo M, Heymsfield SV, et al. (2003) Is percentage body fat differentially related to body mass index in Hispanic Americans, African Americans, and European Americans? Am J Clin Nutr 77, 71-75.

10. Deurenberg P, Weststrate JA \& Seidell JC (1991) Body mass index as a measure of body fatness: age- and sex-specific prediction formulas. Br J Nutr 65, 105-114.

11. Gallagher D, Visser M, Sepulveda D, et al. (1996) How useful is body mass index for comparison of body fatness across age, sex, and ethnic groups? Am J Epidemiol 143, 228-239.

12. Gallagher D, Heymsfield SB, Heo M, et al. (2000) Healthy percentage body fat ranges: an approach for developing guidelines based on body mass index. Am J Clin Nutr 72, 694-701.

13. Jackson AS, Stanforth PR, Gagnon J, et al. (2002) The effect of sex, age, and race on estimating percent body fat from BMI: the Heritage Family Study. Int J Obes 26, 789-796.

14. Ogden CL, Carrol MD, Curtin LR, et al. (2006) Prevalence of overweight and obesity in the United States, 1999-2004. JAMA 295, 1549-1555.

15. Flegal KM, Graubard BI, Williamson DF, et al. (2005) Excess deaths associated with underweight, overweight, and obesity. JAMA 293, 1861-1867.

16. Flegal KM, Graubard BI, Williamson DF, et al. (2007) Causespecific excess deaths associated with underweight, overweight, and obesity. JAMA 298, 2028-2037. 
17. Fontaine KR, Redden DT, Wang C, et al. (2003) Years of life lost due to obesity. JAMA 289, 187-193.

18. Stevens J, Plankey MW, Williamson DF, et al. (1998) The body mass index-mortality relationship in white and African American women. Obes Res 6, 268-277.

19. Manson JE \& Bassuk SS (2003) Obesity in the United States: a fresh look at its high toll. JAMA 289, 229-230.

20. Lohman TG \& Chen Z (2005) Dual energy X-ray absorptiometry. In Human Body Composition, chapter 4, pp. 63-78 [SB Heymsfield, TG Lohman, Z Wang and SB Going, editors]. Champaign, IL: Human Kinetics.

21. Rabe-Hesketh S \& Skrondal A (2008) Multilevel and Longitudinal Modeling Using Stata, 2nd ed. College Station, TX: Stata Press.
22. Twisk J (2003) Applied Longitudinal Data Analysis for Epidemiology: A Practical Guide. Cambridge, UK: Cambridge University Press.

23. StataCorp (2007) User's Guide: Release 10. College Station, TX: StataCorp LP.

24. West BT, Welch KB \& Gatecki AT (2007) Linear Mixed Models. New York: Chapman \& Hall/CRC.

25. Stanforth PR, Jackson AS, Green JS, et al. (2004) Generalized abdominal visceral fat prediction models for black and white adults age 17-65: The Heritage Family Study. Int J Obes 28, 925-932.

26. Reich D, Patterson N, Ramessh V, et al. (2007) Admixture mapping of an allele affecting interleukin 6 soluble receptor and interleukin 6 levels. Am J Hum Genet 80, 716-726. 